\title{
2
}

\section{DE LA TEORÍA A LA PRÁCTICA EDUCATIVA EN IGUALDAD}

\section{FROM THEORY TO EDUCATIONAL PRACTICE IN EQUALITY}

\author{
Ana Vega Navarro \\ amvega@ull.edu.es \\ Sara García Cuesta \\ sagarcia@ull.es \\ Bernardo A. Candela Sanjuan \\ bcandels@ull.es \\ José Diego Santos Vega \\ dsantos@ull.es \\ Matilde Díaz Hernández \\ macadine@ull.es \\ Esther D. Torrado Martín-Palomino \\ estorra@ull.es
}

Universidad de La Laguna, España

https://doi.org/10.25145/b.innovaull.2019.002 


\section{RESUMEN}

El grupo consolidado de innovación educativa Feminario ULL lleva varios años trabajando por incorporar la perspectiva de género en la docencia universitaria. Durante el curso 2017/2018 ha continuado con esta tarea, cuyos objetivos han sido: a) realizar un análisis de necesidades desde cada asignatura implicada en el proyecto; b) provocar la reflexión en el alumnado sobre las condiciones de las mujeres y los obstáculos para la igualdad real en la sociedad española e internacional; y c) diseñar estrategias y acciones para hacer visible los estereotipos sexistas tanto dentro como fuera del aula en busca de igualdad social. Este texto muestra los esfuerzos dedicados a esta labor y algunos de los resultados obtenidos, mostrando cuáles han sido las estrategias metodológicas que han permitido dentro del aula que el alumnado reflexione e incorpore la perspectiva de género tanto a sus vidas cotidianas como a sus prácticas académicas, y también fuera del aula, a través fundamentalmente de diferentes acciones de divulgación que han llevado a un público amplio debates e ideas.

PALABRAS CLAVE: Innovación educativa; género; inclusión; proceso enseñanzaaprendizaje; Universidad.

\section{ABSTRACT}

The group of educational innovation «Feminario ULL» has been working for several years to incorporate the gender perspective in university teaching. During the course 2017/2018 has continued with this task, whose objectives have been: a) perform a needs analysis from each subject involved in the project; b) provoke reflection in the students on the conditions of women and obstacles to real equality in Spanish and international society; and c) design strategies and actions to make visible the sexist stereotypes both inside and outside the classroom in orden to get social equality. This text shows the efforts dedicated to this work and some of the results obtained, especially the strategies that have made students reflect and incorporate the perspective gender into their daily lives as well as into their academic practices, and also outside the classroom, through different dissemination actions that have led to a broad public debate and ideas.

KEYWORDS: Educational innovation; gender; Inclusion; Teaching-Learning Process; University.

\section{INTRODUCCIÓN}

Reflexionar y profundizar cuestiones relativas a los procesos de aprendizaje entre el alumnado universitario es una obligación educativa en el siglo XXI. El entorno universitario debe incorporar la perspectiva de género a las instituciones educativas con el objetivo de trabajar para erradicar las desigualdades de género, así como otros tipos de desigualdad presentes en nuestras sociedades, tanto en contenidos y actitudes como en formas de organización. Así, este trabajo se centra, de manera prioritaria, en la integración de igualdad e inclusión con distintas acciones que se producen en el aula, como parte activa y transversal de algunas 
asignaturas o materias de diferentes titulaciones de la Universidad de La Laguna, y también fuera del aula, buscando extender la influencia de la universidad más allá de sus paredes.

El procedimiento que hemos venido siguiendo durante los últimos años ha consistido en partir de un análisis de necesidades desde cada asignatura implicadas en el proyecto. Se trata de provocar en el alumnado la reflexión sobre una perspectiva de igualdad e inclusión en la docencia con las actividades propuestas, así como plantear y diseñar estrategias y acciones para hacer visible los estereotipos bajo la perspectiva de género, siendo conscientes de que estos estereotipos inundan todavía el entorno social y, por extensión, el aula universitaria.

La metodología se ajusta a la secuencia cíclica propia de los procesos de innovación y mejora: análisis de la realidad y determinación del ámbito de mejora, planteamiento de objetivos y acciones, desarrollo y evaluación de la implementación de las mejoras en las asignaturas en los distintos grados en donde se ha intervenido.

Se han desarrollado diferentes actividades en las asignaturas participantes, unas encaminadas a introducir en el ámbito de aula la perspectiva de género con la finalidad de que se produzca el efecto concreto de un replanteamiento de las labores profesionales a las que conducen los distintos Grados que desarrolla el alumnado; y otras actividades están pensadas para la divulgación para un público más amplio, más allá del aula universitaria, de los trabajos feministas actuales.

Durante el año académico 2017-2018, las acciones implementadas se han centrado más específicamente en la labor de difusión y sensibilización, si bien se han mantenido y desarrollado las actividades específicas en las aulas, con diversas asignaturas del profesorado implicado.

\section{ACCIONES PARA FAVORECER LA IGUALDAD EN EL AULA UNIVERSITARIA.}

Las asignaturas de Didáctica General: enseñanza y currículo y Relación Familia Escuela han sido impartidas por la profesora Ana Vega Navarro. Ambas asignaturas corresponden al Grado de Pedagogía, y han sido desarrolladas durante el primer cuatrimestre. Didáctica general: enseñanza y currículo cuenta con 56 estudiantes (42 mujeres y 14 hombres) y corresponde al $2 \circ$ Curso de la carrera. Mientras que Relación Familia Escuela acoge a 48 estudiantes (40mujeres y 8 hombres)de 4 을 de curso. Las distintas actuaciones propuestas dentro del proyecto en las asignaturas señaladas en las Guías docentes, se llevaron a cabo en varias acciones desde la perspectiva de Género:

Feminicidio 2017: Esta actividad se realizó en la asignatura Relación Familia Escuela, con la intención de visibilizar el tema de violencia machista en el ámbito doméstico, acoso... en busca de causas, motivos, además 
de llevar una reflexión sobre el tema con el alumnado: prevención, cómo actuar en esos casos... Para ello invitamos a dar una charlaTaller a Dolores Delgado Rodríguez (Servicio Insular de atención Especializada a víctimas de Violencia de Género), que realizó un trabajo con el alumnado sobre los feminicidios producidos durante el año 2017 (Mujeres, niñas y niños). En este trabajo se busca la concienciación social y sensibilización sobre la violencia machista, un problema social de gran envergadura, que tiene decenas de víctimas cada año en España. El alumnado trabajó en la web Feminicidio. net, con las estadísticas y los datos de las víctimas de género. Una vez concienciadas y concienciados, cada estudiante escogió un caso de víctima y realizaron su silueta, con los datos más relevantes del caso (perfiles de mujeres, niñas y niños) con el objetivo de hacerlas visibles todas juntas en un escenario público y académico. La muestra estuvo expuesta del día 20 de noviembre hasta el 4 de diciembre, en el edificio central de la ULL, haciendo coincidir con la fecha del $25 \mathrm{~N}$, día internacional contra la violencia de género.

Exposición de cuentos de Gatas y Gatos: En ambas asignaturas la interiorización y utilización de un lenguaje inclusivo estuvo muy presente. Estaba presente como criterio de evaluación en las guías docentes, y dimos un paso más e intentamos fijarnos en las imágenes masculinas y femeninas en los cuentos señalando número, acciones que aparecen, papel protagonistas, secundarios de las mujeres y niñas. Para ello, pusimos en marcha la exposición de Cuentos de gatos y gatas. La exposición nos dio la oportunidad de trabajar distintos tipos de familia y competencias de responsabilidad, trabajo en grupo, en equipo etc. Asimismo, favoreció la colaboración del alumnado (chicos y chicas) en el montaje de la exposición, selección de cuentos, contacto con distintos colegios para dibujos de gatas y gatos.

Los cuentos siguen contando. Cuentos para la igualdad: Los cuentos tradicionales han sido concebidos o recopilados en un momento histórico en el que no se planteaba la igualdad. Contienen, por tanto, rasgos sexistas muy evidentes. Como no se trata de reescribir los cuentos, ya están en nuestras manos, es necesario que hagamos conscientes esos rasgos, para poderlos contrarrestar. Es necesario que, además de la voz, recuperemos la imagen: los libros ilustrados, que son un soporte esencial en las escuelas, en las casas, enseñan a los niños que deben ser activos y dinámicos; deben tener valor y un protagonismo superior a las niñas. Las imágenes retratan a las niñas como pasivas, limpias, ordenadas, subordinadas; deben ser amables y dóciles. Muchas veces las imágenes estereotipadas son mutilantes para los niños y empobrecedoras para las niñas. Los papeles rígidos aprisionan y modifican la personalidad. La obediencia al modelo clásico, imitación a modelos masculinos. Los niños, sin referentes a un modelo femenino, no conciben que las mujeres puedan jugar un 
papel diferente al que se muestra en los libros ilustrados: doméstico y contenedor. La exposición quiere romper con todo esto y mostrar alternativas, dando la oportunidad de conocer diferentes formas y romper con los estereotipos clásicos. Esta exposición fue el resultado de la acción colaborativa del alumnado de la asignatura y se mostró al público entre los días 7 al 23 de marzo, en coincidencia con la semana del 8 de Marzo, conmemorativa del Día Internacional de las Mujeres. Criterios de evaluación y visibilidad a las autoras académicas femeninas: En ambas asignaturas también tratamos de visibilizar las autoras académicas y poner nombres completos, evitando sustituir los nombres por las iniciales para acercar el conocimiento generado por las mujeres, que la mayoría de las veces queda en segundo plano o se presupone en sus iniciales que son varones. En las clases prácticas de las dos asignaturas la perspectiva de género fue central: desde la asignatura Relación Familia Escuela tuvimos oportunidad de tener varios invitados compartiendo diferentes experiencias. En la asignatura de Didáctica General: enseñanza y currículo tuvieron que realizar una propuesta didáctica y diseñar una Unidad Didáctica o Situación de Aprendizaje en perspectiva de género. Este curso académico el tema propuesto fuel@s refugiad@s de Siria. El alumnado se centró en el análisis de las mujeres en los campos de refugiad@s, con fenómenos exclusivamente asociados a ellas como son las violaciones y la inseguridad física, sexual, de salud. Asimismo, sus necesidades específicas como madres en estos lugares. Este trabajo removió mucho en el alumnado, siendo muy bien valorado.

Todas estas actividades que han tratado de impregnar los contenidos, análisis de tareas, consecución de competencias desde la perspectiva de género, han sido muy enriquecedoras, tanto para el alumnado como para la profesora. Los resultados académicos positivos de la asignatura Relación Familia Escuela ha sido de más del $95 \%$ y Didáctica General del $82 \%$. Estas actividades y el tema propuesto han sido significativamente valoradas por el alumnado, que ha dado pasos en la visibilización de los estereotipo de género y las conductas sexistas y machistas. Esto lo valoramos muy positivamente en la formación de futuros profesionales de la enseñanza y futuros ciudadanos y ciudadanas, madres y padres. En todas las actividades propuestas las y los estudiantes han colaborado con una disposición, interés, ganas e implicación de una forma que anima a seguir trabajando en estos temas desde las asignaturas. Han invertido bastantes horas extras en colaborar en las exposiciones, asistir a diferentes actividades y eventos. Esto no quiere decir que esté todo solucionado ni mucho menos, tenemos y tendrán que seguir reflexionando y formándonos sobre este tema.

La asignatura "Sociología de la Población», en tercer curso del Grado de Sociología, es una asignatura troncal obligatoria de 6 créditos, que se desarrolla en el primer cuatrimestre de cada curso académico, 
impartida por la profesora Sara García Cuesta. En este curso 2017-2018 participan en la asignatura 29 estudiantes de Sociología, 16 hombres y 13 mujeres de edades comprendidas entre los 21 y 30 años, de diferentes procedencias (diferentes islas del archipiélago canario, tres estudiantes Erasmus y dos estudiantes de otras universidades peninsulares).

En el marco de esta asignatura, la profesora ha realizado cuatro acciones vinculadas directamente a su participación en el Proyecto de Innovación Docente «Feminario ULL» para el curso 2017-2018. A continuación, se exponen brevemente las cuatro actividades, con una pequeña valoración de su desarrollo, resultados y propuesta de mejora.

\section{Protocolo para la elaboración Y entrega de documentos:}

\section{TRABAJOS, INFORMES, COMENTARIOS DE TEXTO, ANÁLISIS PARCIALES, REFLEXIONES.}

El Protocolo, disponible en el aula virtual de la asignatura, fue presentado en clase el día inaugural de esta y, en principio, acogido sin dudas. Aprovechando la exposición y los links a los que remite el escrito, se presentaron los instrumentos para la igualdad de los que dispone el alumnado en el marco institucional (UIG-ULL, Representantes de igualdad de los centros, Protocolo contra el acoso, Plan de lgualdad). Posteriormente, en la entrega de los trabajos durante la evaluación continua, el alumnado pudo notar, reflejado en las calificaciones y comentarios valorativos asociados a esta, que la vigencia del protocolo era real y quedó sorprendido por ello, y yo porque estuvieran sorprendidos/as, haciéndoles notar de facto el ejemplo de devaluación académica de todas las cuestiones relacionadas con la perspectiva de género, de manera inconsciente.

De esta forma, tanto el protocolo como, en general, el uso y aplicación de lenguaje no sexista dio lugar a un debate en clase, que permitió ampliar los conocimientos y sensibilizar al alumnado más hostil al uso de genéricos no masculinos. En este debate participaron estudiantes con distintas posturas, permitiendo la aclaración de dudas, facilitando ideas para el uso inclusivo de la lengua escrita, entre toda la clase. Asimismo, pudieron conocer de primera mano la guía de lenguaje no sexista de la ULL, que muchos de ellos no habían consultado en primera instancia, al desconsiderar inicialmente el valor del protocolo de normas para la presentación del trabajo. Entiendo este debate como algo muy positivo, pues abrió un espacio para la discusión e información en un ambiente académico y de tolerancia. Permitió llegar a acuerdos y sensibilizar sobre la relevancia de evitar los prejuicios de género en los análisis sociodemográficos y, más en general, en el lenguaje. Presento el Protocolo completo (junto con las normas sobre la perspectiva de género hay otras más generales) elaborado en el contexto del Feminario ULL, y adaptado posteriormente a la asignatura que imparto, para propiciar su uso en el entorno académico. La propuesta para el próximo curso es realizar una versión digital del protocolo, mejorada y con iconografía, para que el instrumento despierte atención y resulte más amable. 


\section{Protocolo para las tareas evaluativas de la asignatura. Cuestiones de Formato:}

- Los documentos llevarán una portada con título, subtítulo, asignatura, curso académico, fecha y nombres y apellido de autoría.

- Los documentos de trabajo se presentarán en Arial 10, con interlineado

1,5 y márgenes $2,5 \mathrm{~cm}$.

- Entre párrafos se dejará un espacio libre.

- Los títulos principales se pondrán en negrita y Arial 11. Los conceptos clave también podrán ser enfatizados con negrita.

- El entrecomillado solo se usará para resaltar frases textuales de entrevistas o textos.

- Las comillas se utilizarán para resaltar elementos o palabras significativas.

- Las clasificaciones se realizarán con letras o con números: a) b) c) ó 1) 2) 3) (u otros formatos).

- Se realizará un índice con hipervínculo a cada capítulo (tabla de contenido).

- Eviten dejar frases o párrafos sin terminar utilizando los tres puntos (...). Sustituir por "etc." ó por "entre otras".

- Las citas con comillas (literales) de más de tres líneas se colocan en párrafo aparte, en cursiva y con tabulación especial. Es obligatorio que tras el párrafo se cite la autoría, fecha de edición y página (Ej: Carrasco, Cristina: 2003, 54). También cuando se trata de resumen de ideas de otras autoras o autores.

- En la elaboración de cuadros, gráficos y mapas con estilo homogéneo en lo posible, Siempre se indicará su numeración (gráfico 1, tabla 4, esquema 2 , etc.), título, año, población, unidad de medida (arriba) y la fuente de información completa y año (abajo).

- Los paréntesis $(32,5 \%)$ se emplearán, sobre todo, para expresiones numéricas o relacionadas. En estas expresiones numéricas se usarán comas para separar decimales y se indicará la unidad (\%, \%0, etc.). - Realicen una revisión ortográfica y gramatical final. Las faltas ortográficas y gramaticales restan, cada una, un punto a su trabajo. En caso de ser más de 5, el trabajo estará suspendido.

\section{Protocolo para las tareas evaluativas de la asignatura. Cuestiones narra- TIVAS Y DE CONTENIDO:}

- En la elaboración de cualquier texto se tendrá en cuenta la perspectiva de género, procurando utilizar términos genéricos que incluyan ambos sexos (alumnado, seres humanos, juventud, personas inmigrantes, vecindad, ciudadanía, etc.). Esta consideración es especialmente relevante en los análisis poblaciones: cuando decimos hombres, nos estamos refiriendo siempre al sexo masculino.

- En caso de que los análisis incluyan la relevancia de efectos, causas, impactos, volúmenes diferenciados entre ambos sexos, deberán 
quedar plasmados en tablas, gráficas y análisis, cuidando de que el lenguaje de estos análisis diferenciales distingan claramente hombres y mujeres, sin asumir el masculino genérico como norma, pues este impide reconocer cuándo se está hablando de unas y otros.

- Para cuidar el lenguaje, procurando su uso no sexista y adecuado al a expresión correcta de los significados de referencia, pueden encontrar materiales de referencias en el siguiente enlace: http://www.igualdad. ull.es/guias $1 . h t m l$. Además, tienen la guía del lenguaje no sexista de la ULL-ULPGC: https://acceda.ulpgc.es/bitstream/10553/18689/1/ Protocolo_Lenguaje_no_sexista_ULPGC.pdf

- Incluir los nombres de las personas con un apellido al menos, evitando aportar derivaciones de los mismos. Eviten Don Pepe, Juanín, Jaime L., etc. En las autorías españolas, citen los dos apellidos, en caso de que estén disponibles, para adecuarse a la perspectiva de género. - Asimismo, los nombres propios de autoría se visibilizarán, evitando siglas y usando el nombre completo. Ej: Sassen, Saskia; Sennett, Richard. En caso de dos apellidos: Sara García Cuesta o García Cuesta, Sara (no S. García, ni García, S.).

- Cuidar al máximo la expresión de la toponimia y la referencia nombres propios: San Cristóbal de La Laguna, Santa Cruz de Tenerife, Área Metropolitana de Tenerife, La Cuesta, La Candelaria, Barranco de Santos, Montaña de Guerra, Calle Narciso de Vera, etc.

- Las ideas que no son propias, aunque no se copien literalmente, deben tener su reconocimiento para no ser plagio. Cuando hagan resúmenes de ideas de otras personas, utilizadas para sus reflexiones, deben citar también al autor o autora. (Ej: la imposibilidad de estrategias a largo plazo en las sociedades líquidas hace que nuestras vidas se definan por la precariedad y la incertidumbre. Se trata de actualizarse rápidamente ante cambios múltiples y constantes para no quedar excluidos (Bauman, Zigmunt, 2007).

- Procuren no repetir con reiteración los mismos términos en el párrafo, para lo que se recomiendo la utilización de un diccionario de sinónimos. Y leer cada día para entrenar el estilo.

- Siempre se añadirá un anexo bibliográfico al final de cada escrito, con la citación completa de cada texto utilizado. Las nuevas normas APA también incluyen como citar textos e información desde internet. Ver http://www.ull.es/view/institucional/bbtk/Referencias_normas_APA/es.

- Es muy importante revisar los textos antes de remitirlos. Se valörará su cuidada redacción y elaboración, devolviéndolos a sus responsables siempre que observe carencias significativas, sobre todo relacionadas con el incumplimiento de este protocolo.

Divulgación en el aula virtual de la asignatura de la página oficial de la Unidad de Igualdad de Género de la ULL, presentando dicha unidad y las herramientas que contiene (observatorio, documentación sobre PG, acciones 
de sensibilización y prevención de desigualdades y violencias de género). Esta charla de 15 minutos se realizó el día de la presentación del funcionamiento y estructura del aula virtual de Sociología de la Población, al inicio del curso.

Actividad para visibilizar científicas en los estudios sociodemográficos. Todo el alumnado en evaluación continua o final fue invitado a desarrollar un ejercicio cuya valoración se reconoce con un punto en la nota final. La actividad consiste en generar un blog o app digital sobre científicas en los estudios sociodemográficos, con información bibliográfica, principales aportaciones e imágenes y otros recursos digitales. Para ello se sugirió la herramienta app gratuita Mobincube.com por su sencillez de uso. Este ejercicio se propone desde el aula virtual, mencionando que se trata de una propuesta en el marco del Programa de Innovación Docente 2017, para promocionar la perspectiva de género en la ciencia. La acción, que fue bien recibida el pasado año de implementación, este año no ha contado con participantes activos. Si bien, un alumno y una alumna manifestaron su interés, finalmente abandonaron la actividad, explicando que las continuas evaluaciones de cada asignatura imposibilitaban realizar ningún trabajo extra y voluntario. Es una reflexión importante sobre la forma en que el exceso de evaluación forzosa está impidiendo los espacios de creatividad y aprendizaje más autónomo.

El alumnado de la asignatura participó en las Jornadas de Migraciones, Género y DDHH en el marco del Campus América, el 17 de Octubre de 2017. Además de la asistencia y participación activa en las sesiones, los y las estudiantes realizaron una reflexión crítica por escrito sobre los contenidos expuestos en dichas jornadas. Aunque estas jornadas eran un añadido a las clases, 17 estudiantes se animaron a seguirlas $(9$ mujeres y 8 hombres) y realizaron un posterior debate en clase sobre los contenidos de estas jornadas relacionados con las temáticas de la asignatura (movimientos de población, especialmente), valorando muy bien su experiencia en estas sesiones.

La asignatura de Psicología Social para el Trabajo social II, impartida por la profesora Matilde Díaz Hernández, corresponde al segundo curso del Grado de Trabajo social y ha sido impartida durante el $2 \circ$ cuatrimestre. En la asignatura de Psicología Social para el Trabajo Social II están matriculados 102 alumnos/as, de los cuales han asistido de forma regular y participado en las actividades propuestas en esta asignatura 90 alumnos/as, siendo el $80 \%$ mujeres y el $20 \%$ hombres. Durante las sesiones prácticas, que se imparten 2 horas a la semana, y las Tutorías Formativas (TAF), asignadas dos horas en el cuatrimestre, se llevaron a cabo tres propuestas de actividades.

Como propuesta de actuación dentro del proyecto y ajustando los contenidos a impartir en la asignatura ya establecidos en la Guía docentes, se llevaron a cabo 3 actividades desde la perspectiva de Género:

Elaboración de una escala de estereotipos: una aproximación. Esta actividad se enmarcó dentro de las técnicas de toma de decisiones y consenso. La actividad consta de distintas fases: a) Tras explicar este tipo de técnica, la tarea a realizar en pequeños grupos, consistía en elaborar una escala tipo Likert, para especificar conductas estereotipadas 
en cinco ámbitos: Trabajo, Familia, Medios de comunicación, Lenguaje y Escuela/Universidad; b) Una vez elaboradas las escalas, se repartieron aleatoriamente entre los pequeños grupos de trabajo para que fuesen revisadas y contestadas por cada grupos y c) Se realizó una reflexión sobre las conductas que se podían observar a través de cada escala en los distintos ámbitos y se corrigieron aquellos ítems que no estaban bien definidos, para llegar a un consenso grupal.

Para la elaboración de este instrumento, se recogieron los resultados en 6 ítems por cada ámbito. La mayoría de los grupos elaboraron las escalas tipo Likert con una escala de valoración del 0 al 5. Los/as alumnos/as definieron qué es para ellos y ellas un estereotipo de género, identificando estereotipos en los cinco ámbitos que se les han solicitado. Siendo el ámbito del lenguaje el que manifiestan como el más complejo de señalar. Finalmente, han mostrado interés por el tema y algunos grupos han seleccionado estos contenidos como tema principal de su trabajo, para el desarrollo de la dinámica por grupos presentanda en clase, al final de la asignatura y como actividad evaluadora de la misma.

Día Internacional de la Mujer 8-Marzo: Las clases prácticas de esta asignatura consistieron en dar a conocer distintas técnicas o dinámicas para trabajar en grupo. Con motivo al día Internacional de la Mujer el 8 de marzo, se trabajó a través de un libro interactivo el sentido y las causas por la cual se realizaba una huelga en la fecha señalada y su conmemoración anual. También se realizó, en pequeños grupos, el juego del trivial feminista con el propósito de aclarar la relevancia de la fecha señalada.

En la Tutorías Formativas (TAF): Por medio de estas tutorías, se pretendió realizar una actividad sobre la elaboración de un informe teórico común, cuyos temas a determinar por el alumnado, abordaronGénero y Liderazgo. Siendo parte del mismo el aprender a realizar un informe teórico según el formato APA, practicar la búsqueda bibliográfica en bases científicas, familiarizarse y aprender la lectura de artículos científicos y por último, aprender y consolidar diferentes conceptos de la investigación en psicología social. En los temas trabajados en estas tutorías, se recogen de manera general los siguientes aspectos: el papel de las mujeres en la política, desigualdad de género en el marco profesional, posición de las mujeres en cargos directivos, estilos de liderazgo entre hombres y mujeres, techo de cristal en el ámbito científico, etc.

Los estereotipos visibles y, a veces no tan visibles, son un tema de gran relevancia para el alumnado de esta asignatura. Las actividades han resultado ser bastante dinámicas y las los estudiantes han trabajado con entusiasmo, mostrando su interés por continuar con este tema. Así lo han manifestado con las actividades que han propuesto para trabajar por grupos en clase y como parte de la actividad evaluadora de las prácticas de la asignatura.

En el marco de la asignatura Sociedad, Cultura y Economía, que forma parte del Diploma de Humanidades y Ciencias Sociales de la EUPAM (Estudios Universitarios para Personas Adultas y Mayores) de la 
ULL, impartida durante el segundo cuatrimestre por el profesor José Diego Santos Vega junto con Javier Marrero Acosta, se han venido desarrollando diversas actividades vinculadas con las intenciones de este Proyecto. Hay que tener en cuenta que esta asignatura está comprometida con el impulso al aprendizaje permanente y continuo a lo largo de toda la vida, por lo que sus implicaciones en la concienciación de las desigualdades de género poseen otras dimensiones y está dirigida hacia un colectivo específico. El alumnado implicado ha sido de aproximadamente 40 mujeres y hombres, en términos muy equilibrado. El contenido impartido en esta asignatura tuvo presente la perspectiva de género, especialmente en el momento de abordar la temática «Familia, relaciones personales y educación", con el objetivo de evidenciar el desigual e injusto tratamiento a las mujeres en el mercado laboral o las desiguales maneras de afrontar y asumir a las tareas de cuidado.

Finalmente, la asignatura «Sociología de la Educación» en el Primer curso del Grado de Maestro y Maestra en Educación Infantil, impartida por la profesora Esther Torrado Martín-Palomino, tiene un carácter troncal obligatorio de 6 créditos, desarrollados en el segundo cuatrimestre de cada curso académico (grupo 2 de tarde). En este curso 2017-2018 participan en la asignatura 101 estudiantes, 8 hombres y 93mujeres de edades comprendidas entre los 18 y los 24 años, de diferentes procedencias de Canarias.

En el marco de esta asignatura, la profesora ha realizado tres acciones vinculadas directamente a su participación en el Proyecto de Innovación Docente ULL «Feminario ULL» para el curso 2017-2018. A continuación, se exponen brevemente las tres actividades, con una pequeña valoración de su desarrollo, resultados y propuesta de mejora:

Incorporación del análisis de la socialización diferencial de género y sus efectos: brechas de género, feminización de las profesiones, currículo oculto en la educación, mediante la docencia en aula, debates, lecturas y videos. En este marco, se han realizado las siguientes actividades: visualizar las diferencias entre el sexo y el género, desmontando los estereotipos tradicionales de género y analizando el currículo oculto, a través de la visualización del video de Sorkunde y realización de dinámicas participativas para detectar todos los espacios del machismo, visibilizando aquellos especialmente ocultos. Así como de la inclusión en el tema de Socialización y Cultura, de la socialización diferencial de género, para visualizar como la socialización diferencial afecta en las trayectorias personales y profesionales de las mujeres y niñas, desde la socialización primaria en la escuela hasta la secundaria en la incorporación al mercado laboral.

Pautas y criterios para la elaboración de poster, trabajos, comentarios de texto. Se ha realizado un trabajo de orientación y asesoramiento permanente al alumnado que va más allá de las exigencias formales académicas. En ese sentido, se exige en la presentación de trabajos en aula, el lenguaje no sexista y recurrir a fuentes paritarias evitando el androcentrismo académico y su reproducción. 
Realización de un producto colectivo con aplicabilidad práctica de los contenidos aprendidos. El alumnado ha realizado un poster sobre las experiencias escolar a través de tres generaciones, mediante un trabajo cooperativo. Así, han desarrollado competencias de trabajo en grupo, de investigación, tratamiento de la información y de análisis desde la perspectiva de género. El objetivo trató de contextualizar las generaciones, analizando las diferencias de género, clase y procedencia interseccionadas por los distintos momentos geopolíticos. Estos productos serán expuestos al final de curso en la Facultad de Educación para que el resto de la Comunidad Educativa pueda visualizarlos.

\section{ACTIVIDADES GENERALES DE DIVULGACIÓN Y SENSIBILIZACIÓN}

Estas actividades han sido generadas buscando el impacto social, especialmente en el alumnado y profesorado de la Universidad de La Laguna:

Exposición sobre Feminicidios en España. Realizada el 25 de Noviembre de 2017 en ocasión del día contra la violencia de género. La actividad buscó visibilizar en el espacio público académico la terrible incidencia de la violencia machista en la actualidad. En una primera fase, el alumnado buscó información sobre los feminicidios, así como de los asesinatos de sus hijas e hijos, que se han producido hasta la fecha a lo largo del año 2017 en las estadísticas oficiales y en webs especializadas (feminicidio.net): nombres y apellidos, motivos, etc. En esta primera fase se pretendió concienciar y sensibilizar a las alumnas y a alumnos de este problema social. Para ello, también otra fase del desarrollo de la actividad intentó ofrecer información sobre la prevención y los procedimientos de actuación ante casos de violencia machista. Este objetivo incluyó la invitación a Dolores Delgado Rodríguez (Servicio Insular de atención Especializada a víctimas de Violencia de Género), a impartir una charla-taller en el aula. Y por último, se adjudicó al alumnado de la asignatura de Relación Familia-Escuela de cuarto curso del Grado de Pedagogía la tarea de realizar una silveta en negro de las mujeres, niñas y niños asesinadas, aportando la información recogida de cada luctuoso caso, para realizar una exposición en el Edificio Central de la Universidad de La Laguna a partir del 25 de noviembre de 2017 con todas las siluetas agrupadas en el suelo, haciendo visible espacialmente el problema, marcando su relevancia y su vigencia. 


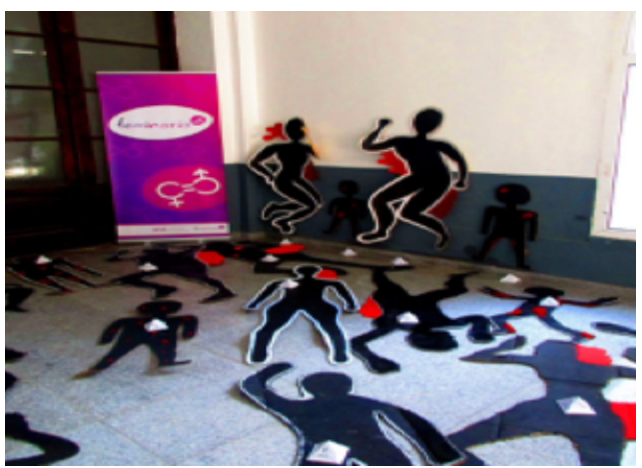

Figura 1. Exposición sobre Feminicidios en España en noviembre de 2017

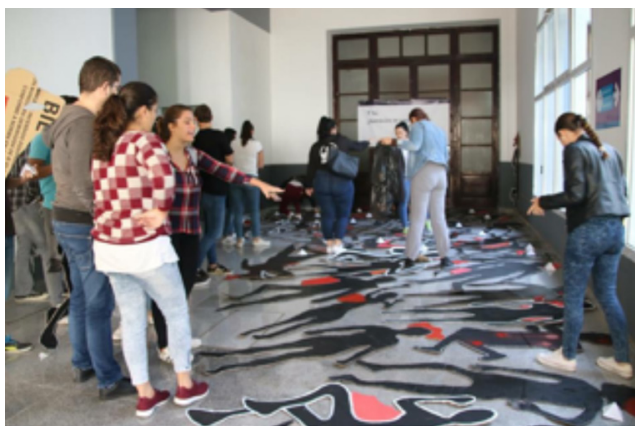

Figura 2. Montaje de la exposición sobre feminicidios en España

Participación en FITVI,17 (Feria Igualdad Tenerife Violeta), durante los días 2 y 3 de diciembre de 2017, en el Parque García Sanabria. El Feminario participó en el stand FITVI '17, informando a los paseantes interesados/as en las actividades que ha venido desarrollando el Grupo de Innovación Educativa consolidado Feminario ULL.

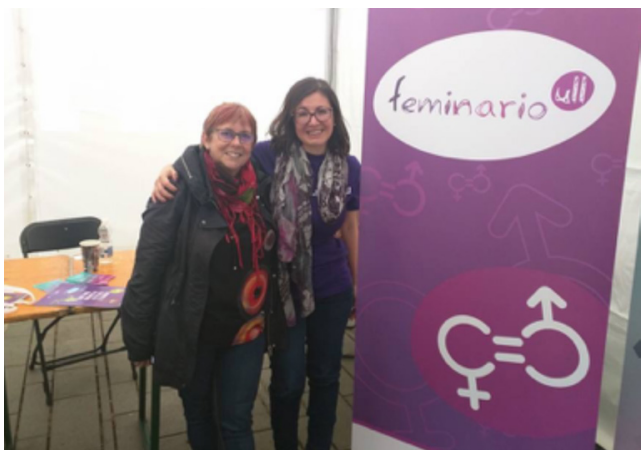

Figura 3. El grupo de innovación educativa «Feminario ULL» participó en diciembre de 2017 en la Feria Igualdad - Tenerife Violeta (FITVI.17). 
Thelma y Louise. Visualización de la película y coloquio-debate, el día 9 de marzo de 2018, en los Multicines Tenerife (C.C. Alcampo, La Laguna). El visionado de la película Thelma y Louise, dirigida por Ridley Scott en 1991, galardonada con el Oscar al mejor guion original, y protagonizada por Geena Davis como Thelma y Susan Sarandon como Louise, permite reflexionar sobre las dificultades cotidianas que sufren las mujeres (acoso y agresión sexuales, violencia machista, desempleo, precariedad laboral, etcétera), así como evidenciar su vigencia en el tiempo, ya que han pasado más de veinticinco años desde su estreno y sigue siendo actual. Posteriormente, se llevó a cabo un debate para tratar los retos que propone la película con expertas en materia de mujer.
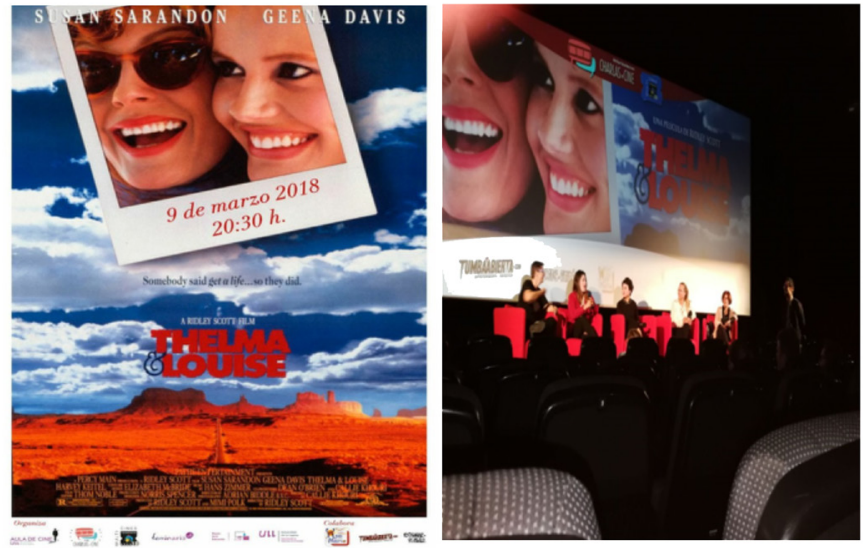

Figura 4. Cartel y fotografía del visionado de Thelma y Louise en Multicines Tenerife, La Laguna.

Exposición Los cuentos siguen contando. Cuentos para la igualdad. Realizado durante los días del 7 al 23 marzo, en el vestíbulo del Edificio Central de la Universidad de La Laguna. En esta exposición se presentan cuentos y juguetes como dispositivos que proponen formas de acción, así como formas de sentir y de pensar, mediadas por diferencias de género, constituyéndonos como sujetos. En la medida que esas historias cuentan, importan, porque narran nuestras historias, narran quiénes somos a través de historias que codificamos y decodificamos y que negociamos en sus ambigüedades. Así, en la exposición se presentan otras historias que no suelen estar tan presentes en el espacio público, como el acoso y la agresión sexual, la diversidad familiar, dando protagonismo a las niñas y mujeres. La exposición tuvo presencia mediática: como muestra un ejemplo en el portal de noticias de la Universidad de La Laguna. 


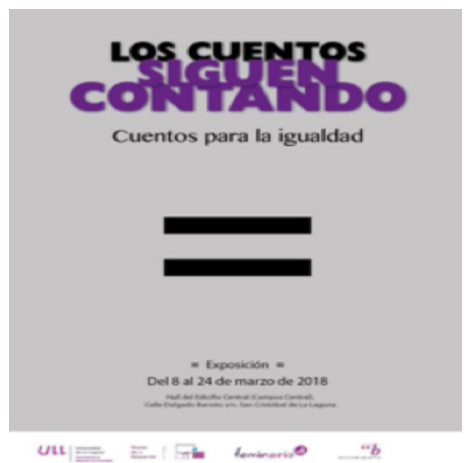

Figura 5. Cartel de la exposición «Los cuentos siguen contando. Cuentos para la igualdad», del 8 al 24 de marzo de 2018.
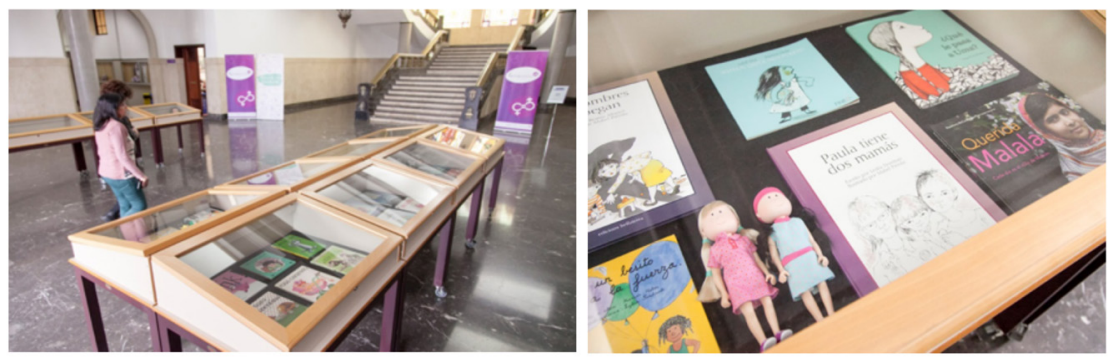

Figuras 6 y 7 . Exposición «Los cuentos siguen contando. Cuentos para la igualdad».

Seminario «La igualdad se aprende, la desigualdad también». Impartido por Nuria Varela (experta en género), durante el día 11 de abril 2018 en el Salón de Actos de la Facultad de Educación (Módulo A) de 11 a 14h. Nuria Varela ejerció de Directora de Gabinete del Ministerio de Igualdad creado en la IX Legislatura, que encabezada Bibiana Aido en el Gobierno presidido por Rodríguez Zapatero. El seminario fue un éxito enorme de afluencia (más de 300 personas), no sólo de alumnado y profesorado universitario sino también de otros niveles, como Bachillerato o Formación Profesional -con alumnado del novedoso ciclo formativo de Técnico Superior en Promoción de lgualdad de Género-e incluso de técnicos y técnicas que desempeñan sus funciones en la administración pública. El contenido del seminario estuvo centrado en los mecanismos cotidianos que en la actualidad nos llevan a perpetuar la desigualdad de género y otras tantas, aunque se haya avanzado en protecciones legislativas, con el objetivo de explicitar esas condiciones y poder trabajar para impulsar la igualdad. El Seminario también tuvo un importante eco mediático: el mismo 11 de abril de 2018, Radio Club Tenerife, de la Cadena Ser, realizó una entrevista a Nuria Varela y en el periódico El Día apareció una noticia al día siguiente, en donde se recogieron las ideas del seminario para avanzar en la igualdad de género. 


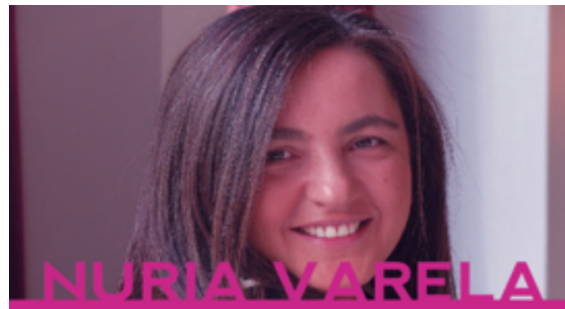

Escritora, experta en violencia de genera, profesora y periodista.

SEMINARIO

La igualdad se aprende, la (des)igualdad, también.

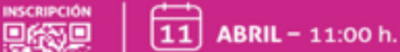

\section{Salón de Actos de la Facultad de Educación. \\ ㅁ. If} San Cristiobal do La Leguna.

teminario $0 \quad \overline{\text { Uu }} \equiv \quad \overline{-}$

Figura 8. Cartel del seminario «La igualdad se aprende, la (des)igualdad, también», impartido por Nuria Varela.

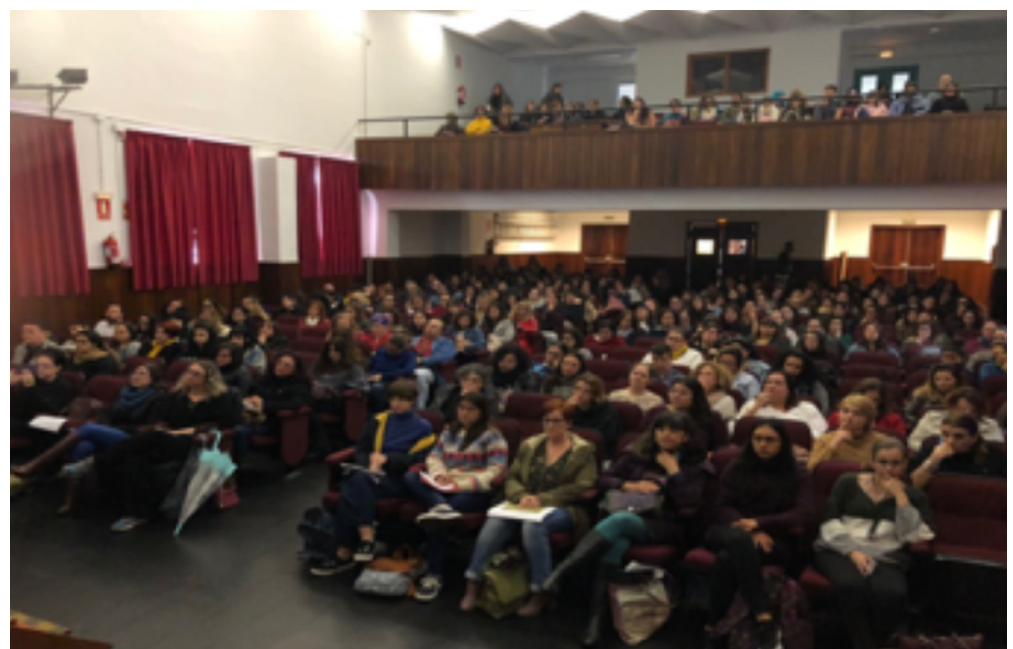

Figura 9. Salón de Actos de la Facultad de Educación de la ULL en el seminario «La igualdad se aprende, la (des)igualdad, también», impartido por Nuria Varela. 


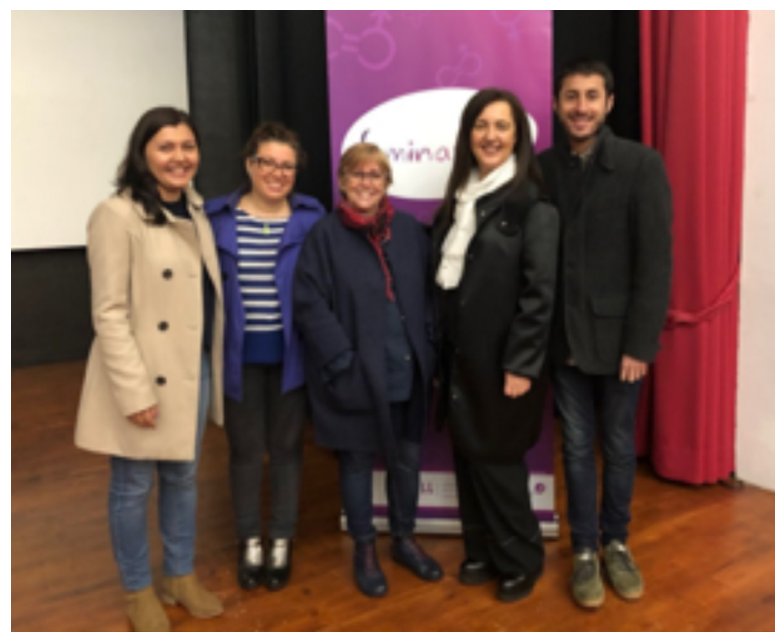

Figura 10. Miembros del grupo de innovación educativa con Nuria Varela

Bar Bahar. Entre dos mundos. Fecha: 8 de mayo de 2018. Lugar: Multicines Tenerife (C.C. Alcampo). Este evento fue anunciado por diferentes guías culturales de Tenerife, como Lagenda. La película Bar Bahar, estrenada en 2016, dirigida por Maysaloun Hamoud, ambientada en Tel Aviv (Israel), trata sobre diversas formas de violencia ejercida contra mujeres palestinas que, enfrentadas a la tradición y al machismo, buscan la libertad. Posteriormente, se llevó a cabo un debate para tratar las vetas que propone la película con expertas en materia de mujer.

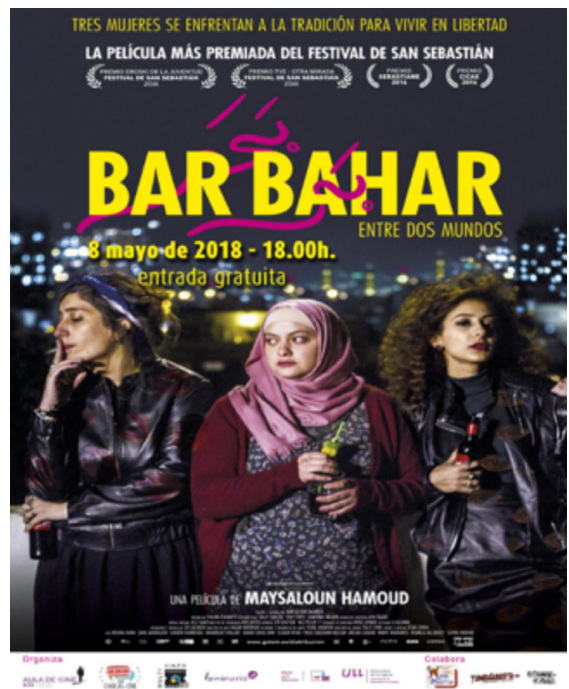

Figura 11. Cartel de la película Bar Bahar en Multicines Tenerife. 


\section{CONCLUSIONES}

Hablar de innovación educativa desde la perspectiva de género supone uno de los mayores retos y desafíos en la educación del siglo $X X I$, toda vez que supone un factor de cambio social educativo que se lo debemos principalmente al movimiento feminista.

Esta innovación parte de una revisión crítica de las culturas androcéntricas y tradicionales, y su objetivo es promover la igualdad, la cohesión y la justicia de género como garantía de equidad.

Pero esta inclusión, para que realmente lo sea, debe garantizar esos cambios desde todos los polos de la organización escolar, esto es, desde los contenidos curriculares, la promoción del lenguaje igualitario, la visibilización de las producciones científicas y docentes de las mujeres, las buenas prácticas, la ciencia con conciencia y como no podría ser de otra manera, la formación permanente del profesorado.

La igualdad y la desigualdad se aprenden a través de las instituciones y agentes de socialización, de ahí la importancia de promover acciones, proyectos y en definitiva políticas de igualdad que no nos lleven a confundir la igualdad real con la legal, pues las leyes per se, no hacen efectiva esas condiciones de igualdad entre mujeres y hombres.

A lo largo de la historia no ha existido la igualdad efectiva entre mujeres y hombres, ya sea porque se ha considerado a las primeras como seres inferiores desde la tradicional teoría de la biología deferencial. Esto ha conferido a las mujeres a espacios y roles tradicionales asignados por cuestiones de sexo, quedando relegadas fuera de los espacios de influencia, poder y visibilidad social. Las investigaciones dentro del feminismo demuestran que esos roles claramente diferenciados entre las mujeres y hombres nada tienen que ver con la biología, es decir, con el hecho de ser biológicamente mujer $u$ hombre, sino que son asignaciones que se construyen socialmente bajo mitos, prejuicios, tradiciones y que se transmiten a través de las instituciones familiares, educativas y las TICS. Por lo tanto, si son construcciones sociales, se pueden destruir, deconstruir y erradicar a través de la sensibilización y el diseño de cambios sociales planificados.

Por eso, las instituciones educativas, entre otras, deben promover la idea de que mujeres y hombres están capacitados igualmente tanto para la vida privada como para la familia, tanto para el trabajo de los cuidados como para el trabajo productivo, pues defender lo contrario significa mantener una diferenciación social y una desigualdad que está al servicio de neutralizar y permitir las prácticas cotidianas de la discriminación.

Por eso, todas las estrategias de innovación con perspectiva de género son pocas. Garantizar una agenda repleta de estrategias con perspectiva de género, constituye la fiel garantía de enseñar en las 
instituciones educativas no sólo valores de igualdad, sino a creer en la igualdad como un derecho inalienable y un elemento fundamental de la paz y convivencia social.

Desde el Feminario ULL no nos conformamos con formar e informar al profesorado sobre los conceptos de igualdad como justicia social, sino que vamos más allá promoviendo estrategias de visibilización e identificación de esas desigualdades que ponen en peligro la convivencia escolar y social. Para las miembras y miembros de este Feminario, aprender a mirar con "gafas violetas» constituye uno de los mayores retos para identificar y combatir las discriminaciones y el sexismo en la escuela y las instituciones de educación superior.

Una vez que hemos aprendido a mirar e identificar las desigualdades y a creer en la igualdad como valor democrático irrenunciable, formar y fomentar las buenas prácticas educativas constituye «el broche final de nuestro trabajo" porque no creemos en una escuela ni segregada ni mixta, sino en un modelo de escuela coeducativa.

\section{REFERENCIAS BIBLIOGRÁFICAS}

Aguilar Carrasco, Pilar (2018): Feminismo o barbarie. Cáceres: La Moderna.

DíAz, Capitolina (2015). La perspectiva de género en la investigación social. En: García Ferrando, Manuel et al (2015). El análisis de la realidad social: métodos y técnicas de investigación (4⿳亠口冋 edición). Alianza Editorial.

ElCHLER, Margrit (1991). Nonsexist Research Methods: A Practical Guide.

HARDING, Sandra (1996). Ciencia y feminismo. Madrid: Ediciones Morata.

Huston, Nancy (2018): Vosotras bellas, Vosotros fuertes. Barcelona: Galaxia Gutenberg.

Ley Orgánica 3/2007, de 22 de marzo, para la igualdad efectiva de mujeres y hombres. BOE» núm. 71, de 23 de marzo de 2007.

Posada Kubissa (2015). Filosofía, crítica y (re)flexiones feministas. Madrid. Editorial Fundamentos.

SalazAr, Octavio (2018). El Hombre que no deberían ser. Barcelona: Planeta

SALDAÑA Díaz, María N. (2006). La educación en igualdad y para la igualdad entre los géneros en el marco normativo de las naciones unidas, la unión europea y constitucional español. Revista de Educación XXI, 201-218.

SANZ, Marta (2108): Monstruas y centauras. Nuevos lenguajes del feminismo. Barcelona: Anagrama.

SubIRATS, Marina (2013). Forjar un hombre, moldear una mujer. Bellcaire d'Empordà, Girona: Aresta.

TORRES RÁmireZ, Isabel (Cood) (2005): Miradas desde la perspectiva de género. Madrid: Narcea. 
UNESCO. (2005). Guidelines for inclusion: Ensuring Access to Education for All. París: UNESCO. Fecha de consulta: Febrero 20 /2012. En: http://unesdoc.unesco.org/ images/0014/001402/140224e.pdf.

Vega Navarro, A., García Cuesta, S., Díaz Hernández, M. C., Espino Espino, E., Calzadilla Medina, M. A., Santos Vega, J. D. y Torrado Martín-Palomino, E. (2018). Una experiencia deinnovación en los procesos de enseñanza-aprendizaje en la Universidad desde la perspectiva de género, De la innovación imaginada a los procesos de cambio. Disponible en: https://riull.ull.es/xmlui/handLE/915/9443.

Varela, Nuria (2017): Cansadas. Una reacción feminista frente a la nueva misoginia. Madrid: Ediciones B, 